\title{
Knockdown of Nrf2 enhances autophagy induced by temozolomide in U251 human glioma cell line
}

\author{
YUAN ZHOU $^{1}$, HAN-DONG WANG $^{1}$, LIN ZHU $^{1}$, ZI-XIANG CONG ${ }^{1}$, NING LI $^{3}$, \\ XIANG-JUN JI ${ }^{1}$, HAO PAN ${ }^{1}$, JIA-WEI WANG ${ }^{1}$ and WAN-CHUN LI $^{2}$ \\ Departments of ${ }^{1}$ Neurosurgery and ${ }^{2}$ Pathology, Jinling Hospital, School of Medicine, \\ Nanjing University; ${ }^{3}$ Department of Neurosurgery, School of Medicine, Second Military \\ Medical University (Shanghai), Jinling Hospital, Jiangsu, Nanjing 210002, P.R. China
}

Received September 4, 2012; Accepted October 18, 2012

DOI: $10.3892 /$ or.2012.2115

\begin{abstract}
Glioblastoma multiforme (GBM) and oxidative stress are closely linked. Oxidative stress affects many signaling pathways and may cause the induction of autophagy. The NF-E2related factor 2 (Nrf2)/Kelch-like ECH-associated protein 1 (Keap1) signaling pathway is the main pathway responsible for cell defense against oxidative stress and Nrf2 is a critical transcription factor related with cancer multidrug resistance. However, the relation between Nrf2 and regulation of autophagy is not well understood. In this study, we used temozolomide (TMZ), which inhibited the viability of GBM cells mainly by inducing autophagic cell death and explored the role of Nrf2 downregulation on autophagy induced by TMZ in GBM cells. In U251-Si-Nrf2 $48 \mathrm{~h}$ after transfection the protein levels of Nrf2 were significantly downregulated, while the protein levels of LC3B-II increased by western blot analysis. Knockdown of Nrf2 also led to a significant increase of autophagic vacuoles and acidic vesicular organelles (AVOs), revealed by transmission electron microscopy (TEM) and acridine orange (AO) staining using flow cytometry. Collectively, these findings demonstrate that knockdown of Nrf2 can enhance the basal level of autophagy in the U251 glioma cell line. Furthermore, after the treatment with TMZ $(100 \mu \mathrm{M})$ for 3 days, the U251-Si-Nrf2 transfected cells showed less viability rate by cell counting kit- 8 (CCK-8) assay and the levels of autophagy increased obviously through analysis of western blot and AO staining using flow cytometry. Taken together, our results suggest that knockdown of Nrf2 may enhance autophagy induced by TMZ in the U251 glioma cell line, which should be further evaluated for novel anticancer activity.
\end{abstract}

Correspondence to: Professor Han-Dong Wang, Department of Neurosurgery, Jinling Hospital, School of Medicine, Nanjing University, 305 East Zhongshan Road, Jiangsu, Nanjing 210002, P.R. China

E-mail: hdwang_nj@yahoo.com.cn

Key words: NF-E2-related factor 2, autophagy, glioblastoma multiforme, temozolomide, chemotherapy

\section{Introduction}

Glioblastoma multiforme (GBM) is the most common and fatal intracranial tumor (1). Till now, multimodality treatments with surgery, radiation and chemotherapy have led to some improvement in prognosis for patients with $\operatorname{GBM}(2,3)$. However, the 5-year survival rate for patients with GBM remains less than $5 \%$ and the median survival time is approximately one year (4).

Multiple lines of evidence indicate that oxidative stress plays an important role in the occurrence and progress of malignant tumors $(5,6)$. Tumor cells themselves can induce oxidative stress. NF-E2-related factor 2 (Nrf2) is considered as a critical regulator of intracellular antioxidants and phase II detoxification enzymes by transcriptional upregulation of many antioxidant response element (ARE)-containing genes (7-10). Nrf2/Kelch-like ECH-associated protein 1 (Keap1) signaling pathway is the main pathway for cell adaptation to oxidative stress. Furthermore, current studies agree that Nrf2 plays a dual role in tumor cells. Apart from its positive role in normal cells, Nrf2 also has its dark side. It is responsible for drug resistance of certain cancer cells (11).

In addition to the activation of Nrf2 response, oxidative stress may cause the induction of macroautophagy (referred to hereafter as autophagy). Autophagy is a self-cannibalization process consisting of the degradation and recycling of organelles and portions in cytosol (12). This process plays a major role in maintaining cellular homeostasis and some reports suggest that the main role of autophagy in cancer cells is cell protection (13-16). However, autophagic cell death could also be initiated in response to sustained intracellular damage caused by hypoxia, chemotherapeutic agents, virus infection, or toxins, especially in gliomas (17). Recent studies found that GBM is resistant to chemotherapy protocols that induce apoptosis but seems to be less resistant to therapies that induce autophagy (18-20). As an effective cytotoxic drug, temozolomide (TMZ) induces significant autophagic cell death in many GBM cells, such as U251 human glioma cell line $(18,21,22)$. Thus, autophagy has potential utility as a target for GBM therapy $(23,24)$. Recently, several experts proposed the hypothesis that there may be cross-talk between Nrf2/Keap1 and autophagy pathways (25). Thus, the aim of the current 
study was to determine whether Nrf2 can influence autophagy in U251 human glioma cell line after TMZ.

\section{Materials and methods}

Reagents. TMZ was kindly supplied by Schering-Plough Co. (Kenilworth, NJ, USA) and was dissolved in dimethyl sulfoxide (DMSO, Sigma Chemical Co., St. Louis, MO, USA) in $100 \mathrm{mM}$ stock solution. TMZ was used at a concentration of $100 \mu \mathrm{M}$. The final concentration of DMSO which did not exceed $0.1 \%$ in the culture medium did not influence cell viability and the expression of the proteins studied as previously described (26). Acridine orange was purchased from Sigma Chemical Co.

Cell culture and transient transfection. Human U251 glioma cells were obtained from American Tissue Culture Colection (ATCC) and cultured in Dulbecco's modified Eagle medium (DMEM, HyClone, IL, USA) with $10 \%$ fetal bovine serum (HyClone) at $37^{\circ} \mathrm{C}$ and $5 \% \mathrm{CO}_{2}$ incubator. The vector pGPH1/ GPF/Neo used for cloning Nrf2 short hairpin RNA (shRNA) was purchased from GenePharma (Shanghai, China). The target sequence was GCAGTTCAATGAAGCTCAACT. The new plasmid was named as Si-Nrf2. Random sequence was used as negative control, which was named as Si-control. Cells were seeded in 6-well plates at $1 \times 10^{6} /$ well and allowed to attach for $24 \mathrm{~h}$ before transfection. Then $\mathrm{Si}-\mathrm{Nrf} 2$ and $\mathrm{Si}$-control were transfected by Lipofectamine 2000 (Invitrogen, CA, USA) according to the manufacturer's protocol. Cells treated with Lipofectamine 2000 alone were set up as blank control, which was named as group Lipo. After incubation at $37^{\circ} \mathrm{C}$ and $5 \% \mathrm{CO}_{2}$ for $48 \mathrm{~h}$, cells were collected.

Ultrastructural analysis of autophagy by transmission electron microscopy (TEM). For the TEM analysis, transfected cells after $48 \mathrm{~h}$ were trypsinized, washed twice with PBS and fixed with ice-cold glutaraldehyde $(2.5 \%$ in $0.1 \mathrm{M}$ cacodylate buffer, $\mathrm{pH}$ 7.4) for $30 \mathrm{~min}$. After washing in PBS the cells were postfixed in $1 \%$ osmium tetroxide $\left(\mathrm{OsO}_{4}\right)$ and embedded in Epon; $0.1 \mathrm{~mm}$ thin sections were stained with uranyl acetate/ lead citrate (Fluka, St. Louis, MO, USA) and viewed in a JEOL JEM-1011 TEM (Tokyo, Japan).

Western blot analysis. To obtain total protein lysates, transfected cells after $48 \mathrm{~h}$ and further treated with or without TMZ $(100 \mu \mathrm{M})$ for 3 days were homogenized in RIPA buffer ( $1 \%$ NP40, $0.5 \%$ sodium deoxycholate, $0.1 \%$ sodium dodecyl sulfate, $1 \mathrm{mM}$ ethylene diamine tetraacetic acid, $1 \mathrm{mM}$ ethylene glycol tetraacetic acid, $1 \mathrm{mM} \mathrm{Na}_{3} \mathrm{VO}_{4}, 20 \mathrm{mM} \mathrm{NaF}$, $0.5 \mathrm{mM}$ DL-dithiothreitol, $1 \mathrm{mM}$ phenylmethane-sulfonyl fluoride, and protease inhibitor cocktail in PBS, $\mathrm{pH}$ 7.4) and centrifuged at $14,000 \times \mathrm{g}$ for $15 \mathrm{~min}$ at $4^{\circ} \mathrm{C}$. Protein concentrations were estimated by Coomassie Plus Protein Assay Reagent (Pierce, IL, USA). Equal amounts of protein from each sample was separated by SDS-PAGE on 8-12\% gels and transferred to polyvinylidene difluoride (PVDF) membrane (Millipore, Bedford, MA, USA). Following incubation with primary antibodies against Nrf2 (Abcam, Cambridge, UK; 1:500), Microtubule-associated protein light chain 3B (LC3B, 1:3000), $\beta$-actin (Santa Cruz Biotechnology, Inc., CA, USA;
1:500), GAPDH (Santa Cruz Biotechnology, Inc.; 1:1000) and peroxidase-conjugated goat anti-rabbit IgG (KeyGen Biotech, Nanjing, China; 1:5000) as the secondary antibody, specific protein bands were visualized using ECL detection system (Amersham Biosciences, Bucks, UK) and exposed radiographic film (Fuji Hyperfilm, Tokyo, Japan). The developed film was digitized using an Epson Perfection 2480 scanner (Seiko Co., Nagano, Japan). The levels of Nrf2 and LC3B were quantified by densitometry using ImageJ program and expressed relative to $\beta$-actin and GAPDH signals, respectively.

Acridine orange (AO) immunofluorescent staining and flow cytometric analysis. Quantification of autophagy by AO staining using flow cytometry was performed as described previously (5). Transfected cells after $48 \mathrm{~h}$ treated with or without TMZ $(100 \mu \mathrm{M})$ for 3 days were stained with $1 \mu \mathrm{g} / \mathrm{ml}$ $\mathrm{AO}$ for $15 \mathrm{~min}$ at $37^{\circ} \mathrm{C}$, trypsinized, washed, and collected in phenol red-free growth medium. Green $(510-530 \mathrm{~nm})$ and red $(650 \mathrm{~nm})$ fluorescence emission from cells illuminated with blue $(488 \mathrm{~nm})$ excitation light was measured with flow cytometer. Depending on their acidity, autophagic lysosomes appeared as the orange/red fluorescent cytoplasmic vesicles, while nuclei were stained green. AO-stained cells were analyzed on a FACSCalibur flow cytometer (Becton, Dickinson and Company, NJ, USA) using FlowJo7.6.1 software (Tree Star, Inc., San Carlos, CA, USA). Autophagy was quantified as a ratio between geomean fluorescence intensity of red vs. green fluorescence (FL3/FL1).

Cell viability assay by cell counting kit-8 (CCK-8) assay. Cell viability was assessed by CCK- 8 assay (Dojindo, Kumamoto, Japan). Cells ( $1 \times 10^{3} /$ well) were seeded to $96-$ well culture plates and cultivated for $24 \mathrm{~h}$ to adhere, then transfected according to the manufacturer's protocol. After $48 \mathrm{~h}$, total medium was changed to $100 \mu \mathrm{l}$ complete culture medium with or without $100 \mu \mathrm{M} \mathrm{TMZ}$ and cells were incubated for a further $72 \mathrm{~h}$. DMSO was used as solvent control. CCK-8 (10 $\mu \mathrm{l})$ was added into every well and incubated for $2 \mathrm{~h}$. Then the OD value was read at $450 \mathrm{~nm}$ using a Bio-Rad ELISA microplate reader (Bio-Rad Laboratories, CA, USA). The viability rate of tumor cells equals (the OD values of treated groups/the OD values of control group) $\mathrm{x} 100 \%$.

Statistical analysis. All experiments were done at least three times. Data were presented as mean \pm SD. The statistical significance of the differences between treatments was assessed using one-way ANOVA followed by Student-Neuman-Keuls test for multiple comparisons. The value of $\mathrm{p}<0.05$ was considered significant. All analyses were performed by SPSS 19.0.

\section{Results}

Transient transfection effect on Nrf2 protein level. To validate the transient transfection effect of two plasmids mentioned above, we tested the protein level of three cell groups $48 \mathrm{~h}$ after transfection, respectively. Si-Nrf2 transfection reduced the Nrf2 protein level compared with transfection of Si-control $(p=0.01)$ and group Lipo $(p=0.003)$. Group Si-control and Lipo showed no difference in Nrf2 protein level (Fig. 1A and B). The efficiency of each transfection was verified by this method. 
$\mathbf{A}$
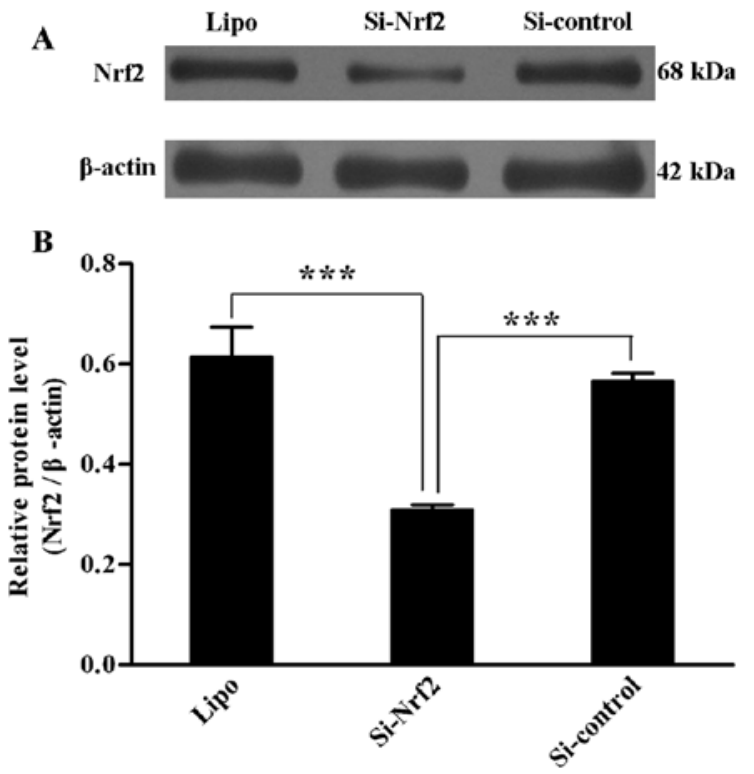

Figure 1. Protein level of Nrf2 analyzed by western blotting $48 \mathrm{~h}$ after transfection. (A) Representative autoradiogram of $\mathrm{Nrf} 2$ expression in each group. $\mathrm{Nrf} 2$ was detected at $68 \mathrm{kDa}$ and the loading control $\beta$-actin at $42 \mathrm{kDa}$. (B) Quantification of Nrf2 protein levels. The graph shows significant downregulation of Nrf2 expression levels in group Si-Nrf2 than group Lipo $(\mathrm{p}=0.003)$ and $\mathrm{Si}-\mathrm{control}(\mathrm{p}=0.01)$. Also, group Si-control and Lipo shows no statistical difference. Data represent mean \pm SD values of three experiments in each group. Asterisks denotes p-value compared with two control groups $\left({ }^{* * * *} \mathrm{p}<0.001\right)$.

Effect of Nrf2 downregulation on the basal level of autophagy in U251 cells. To examine the role of Nrf2 downregulation on the basal level of autophagy in U251 cells, autophagyrelated qualitative and quantitative detection were performed accordingly.

We observed autophagic structures by TEM. The result showed that autophagic vacuoles increased in group Si-Nrf2 compared to group Si-control. Most of the autophagosomes in group Si-Nrf2 contained lamellar structures or residual digested components, whereas tumor cells of group Si-control exhibited few such features (Fig. 2A and B). Also shown were representative images of an autophagolysosome containing undegraded cellular contents (Fig. 2C) and the fusion of a late stage autophagic vacuoles with degraded cellular contents (Fig. 2D).

To quantify the incidence of the basal level of autophagy, we tested the expression of two LC3B forms (LC3B-I and LC3B-II) using western blot analysis. Si-Nrf2 transfection significantly raised the LC3B-II protein level compared with transfection of Si-control ( $<<0.001)$ and Lipo ( $<<0.001)$ (Fig. 3A and B).

In addition, we quantified the presence of acidic vesicular organelles (AVOs) by flow cytometry with AO staining (4). Group Si-Nrf2 had more AVOs (p=0.017) (Fig. 4A-D) in comparison with group Si-control. Collectively, the findings demonstrated that group Si-Nrf2 had a higher level of basal autophagy than control groups.

Effect of Nrf2 downregulation on autophagy induced by TMZ in U251 cells. After treatment with TMZ $(100 \mu \mathrm{M})$ for 3 days, transfected U251 cells were treated similar to the above methods. Western blot results showed that group Si-Nrf2 treated

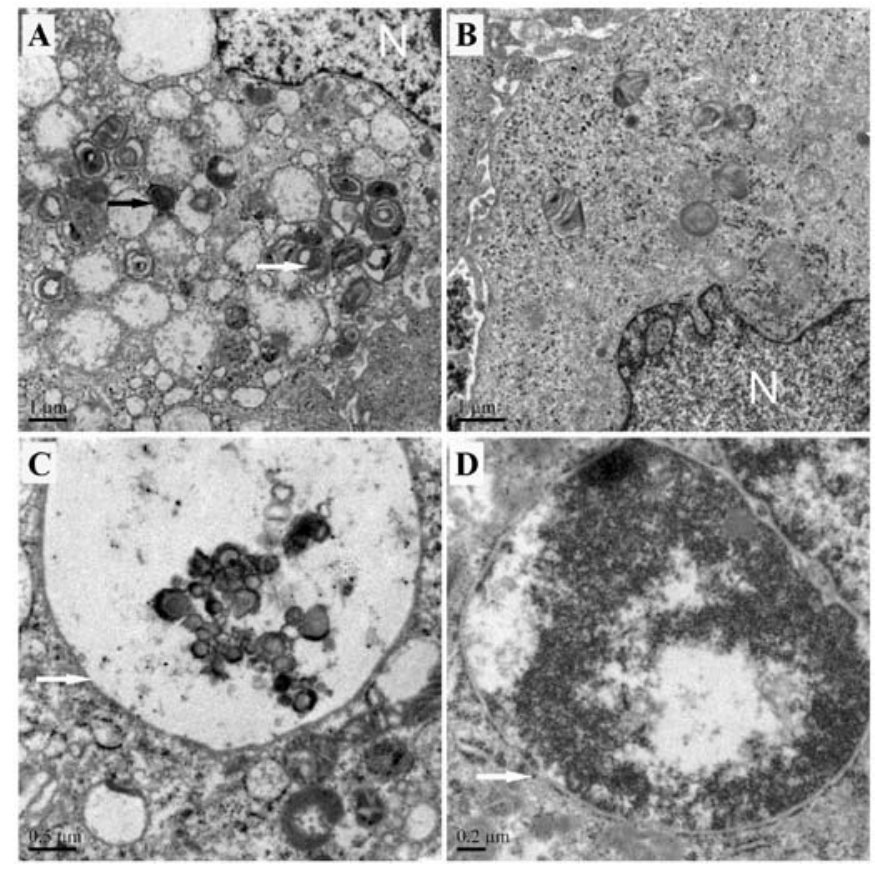

Figure 2. Representative electron micrographs showing the ultrastructure of U251 glioma cells transfected after 48 h. (A) Group Si-Nrf2. A white or black arrow indicates autophagosome or secondary lysosome, respectively. $\mathrm{N}$ indicates nucleus. (B) Group Si-control. N indicates nucleus. (C) A white arrow indicates autophagosome including lamellar structure. (D) Autophagosome including residual digested material (white arrow). Bar (A and B), $1 \mu \mathrm{m} ;(\mathrm{C})$, $0.5 \mu \mathrm{m}$; (D), $0.2 \mu \mathrm{m}$.

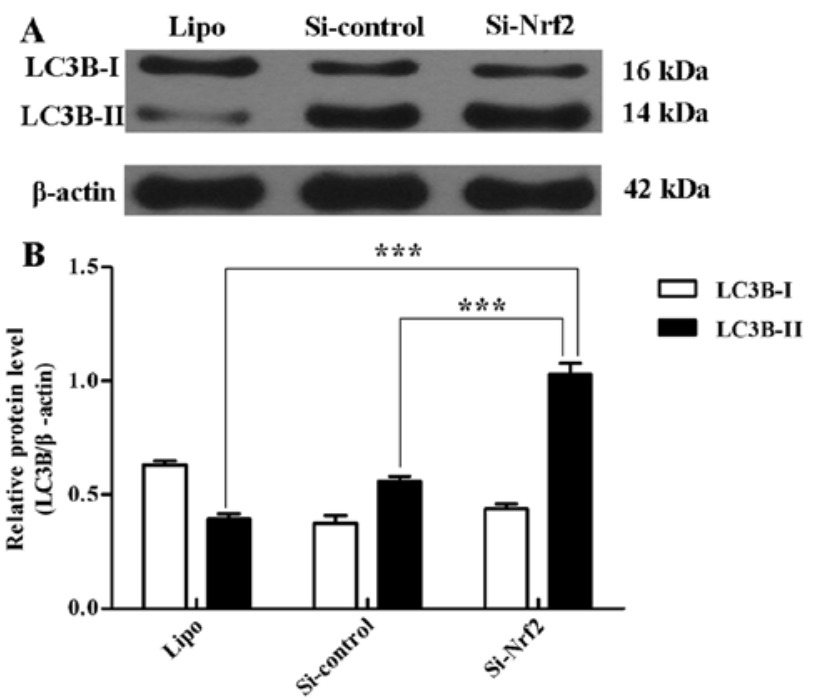

Figure 3. LC3B protein analysis by Western blotting was performed $48 \mathrm{~h}$ after transfection. (A) Representative autoradiogram of LC3B expression in each group. LC3B-I and LC3B-II were detected at 16 and $14 \mathrm{kDa}$, respectively. The molecular weight of internal control GAPDH was $36 \mathrm{kDa}$. (B) Quantification of LC3B protein levels. The graph shows significant upregulation of LC3B-II expression levels in group Si-Nrf2 than other control groups $(\mathrm{p}<0.001)$. Data represent mean $\pm \mathrm{SD}$ values of three experiments in each group. Asterisks denotes p-value between two groups. ${ }^{* * * *} \mathrm{p}<0.001$, group Si-Nrf2 vs. group Si-control and Lipo.

with TMZ raised the protein level of LC3B-II compared with other groups $(\mathrm{p}<0.001)$. In group Lipo, cells treated with TMZ had more LC3B-II expressions than with DMSO treatment $(\mathrm{p}=0.007)$ (Fig. 5A and B). Likewise, there were more AVOs 

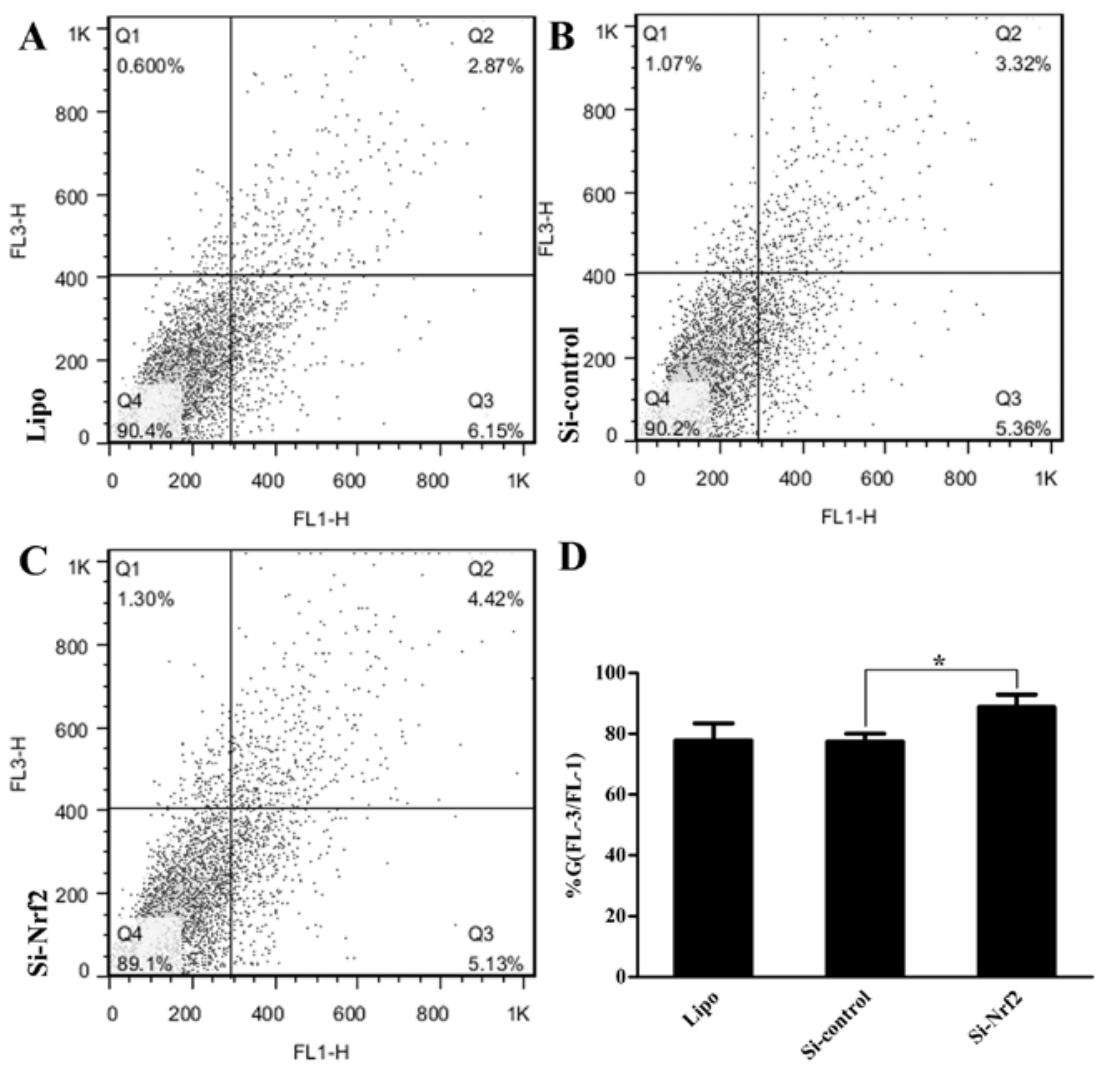

Figure 4. Quantification of AVOs detected by flow cytometry with AO staining in U251 glioma cells transfected after 48 h. (A-C) Representative image for group Lipo, Si-control, Si-Nrf2, respectively. (D) The geomean red:green fluorescence ratio was determined as described in Materials and methods. Group Si-Nrf2 had more AVOs than group Si-control ( $\mathrm{p}=0.017)$. Data represent mean $\pm \mathrm{SD}$ values of three experiments in each group. Asterisks denotes $\mathrm{p}$-value compared with Si-control group ( $\left.{ }^{*} \mathrm{p}<0.05\right)$.

in group $\mathrm{Si}-\mathrm{Nrf} 2$ than other groups $(\mathrm{p}<0.05)$ detected by flow cytometry (Fig. 6A-E).

Effect of Nrf2 downregulation on cell viability in U251 cells after TMZ. To characterize the effect of Nrf2 downregulation on viability of glioma cells after TMZ, we performed CCK-8 assay. Group Lipo treated with TMZ had much less cells than the one treated with DMSO $(\mathrm{p}<0.001)$. After TMZ, cells in group $\mathrm{Si}-\mathrm{Nrf} 2$ showed a decrease compared to those in group Si-control $(\mathrm{p}=0.015)$ and Lipo $(\mathrm{p}=0.007)$ in cell viability. Group Si-control and Lipo showed no significant difference (Fig. 7).

\section{Discussion}

In this study, the results showed that knockdown of Nrf2 significantly enhanced the basal level of autophagy in U251 cells characterized by increased autophagic structures by TEM, elevated expression of LC3B-II at protein level and increased AVOs by flow cytometry. In addition, higher level of autophagy and lower level of cell viability were found in group Si-Nrf 2 than in the other groups after TMZ. To our knowledge, the findings showed for the first time the Nrf2 downregulation enhanced autophagy induced by TMZ in U251 cells.

We evaluated the protein level of Nrf2 after transfection because it reflected the transient transfection efficiency and correlated with the effectiveness of follow-up results. It has been revealed that oxidative stress can be induced by tumor cells. Nrf2 is mutational and overexpressed in many tumor tissues and cell lines $(27,28)$. Nrf2 transcription complex specifically recognizes ARE, which is located in the promoter region of all Nrf2-targeted genes and subsequently initiates the expression of a group of genes. These Nrf2-targeted gene products exert protection for cells from oxidative damage (5). As previously reported, Nrf2 was highly expressed in U251 cells and the transfection efficiency was most predominant at $48 \mathrm{~h}$ after transfection $(5,29)$. Hence, we chose the U251 cells and accessed the transient transfection $48 \mathrm{~h}$ after transfection. Consistent with previous results, $\mathrm{Si}-\mathrm{Nrf} 2$ transfection significantly reduced the Nrf2 protein level compared with transfection of Si-control.

The basal level of autophagy in GBM cells is higher than that in normal cells. Oxidative stress may be the fundamental cause. The basal level of autophagy is vital for the biological activity of tumors. In order to evaluate whether Nrf2 can regulate basal autophagy, TEM, quantification of LC3B-II protein levels and AVOs were performed. The use of TEM is a valid and important morphological method for various autophagic structures $(30,31)$. In this study, a larger number of autophagosomes and autophagolysosomes that correlated with the induction of autophagy were found in group Si-Nrf2 than group Si-control. It is known that LC3B-II is closely associated with the membrane of autophagosomes and AVOs are characteristic of autophagy (32-34). Elevated expression of LC3B-II and number of AVOs reflected that knockdown of Nrf2 caused an increased basal level of autophagy. This indicated that $\mathrm{Nrf} 2$ was responsible for regulating basal levels 




Figure 5. The levels of LC3B-I and LC3B-II expression in transfected cells after $48 \mathrm{~h}$ and further treated with or without TMZ $(100 \mu \mathrm{M})$ for 3 days. (A) Representative western blots. (B) Quantification of LC3B protein levels. The LC3B-II level of group Si-Nrf2 was significantly more than other control groups $(\mathrm{p}<0.001)$. Group Lipo+TMZ had more LC3B-II expressions than group Lipo+DMSO $(\mathrm{p}=0.007)$. Results shown are the means \pm SD of three independent experiments. Asterisks denotes p-value between two groups. ${ }^{* * *} \mathrm{p}<0.001$, group $\mathrm{Si}-\mathrm{Nrf} 2+\mathrm{TMZ}$ vs. other control groups, ${ }^{* *} \mathrm{p}<0.01$, group Lipo+TMZ vs. group Lipo+DMSO.

of autophagy in U251 cells. Our finding was in concordance with previous reports in other tumors (35). Nevertheless, from our point of view, further investigation still needs to be done because Nrf2-responsive genes that stimulate autophagy are not well known.

Earlier studies indicated that TMZ may exert its cytotoxicity mainly by apoptosis pathway, while recent studies investigating the cytotoxic effects of TMZ in malignant gliomas had focused more on autophagy (32). Glioma studies in vitro also showed that autophagy, not apoptosis, was induced by TMZ (19). Autophagy induced by TMZ is important for GBM chemotherapy. Therefore, the present study paid attention to the effect of Nrf2 downregulation on autophagy induced by TMZ. Substantial evidence showed that Nrf2 and apoptosis pathway may cross-talk mediated by the Keap1-binding proteins such as phosphoglycerate mutase 5 (PGAM5), prothymosin $\alpha$ (ProT $\alpha$ ), fetal Alz-50 clone 1 (FAC1), and p62 (SQSTM1) (25). Hence, in order to exclude the role of Nrf2 on apoptosis induced by TMZ, we chose a certain dose and time of TMZ based on previous studies. At this clinically achievable dose $(100 \mu \mathrm{M})$, TMZ induced autophagy, not apoptosis in U251 cells $(19,21,36)$. With TMZ, we observed a significant decrease in cell viability and increase in the level of LC3B-II compared to DMSO. Nevertheless, the difference detected by flow cytometry lacked statistical significance. This may be attributed to the error of operation and poor state of the cells caused by toxicity of TMZ. We speculate that lower cell viability may be connected with higher level of autophagy induced by TMZ, which may be caused by autophagic cell death induced by sustained TMZ treatment. Our data suggested that Nrf2 limited the extent of autophagy induced by TMZ, which was in agreement with the
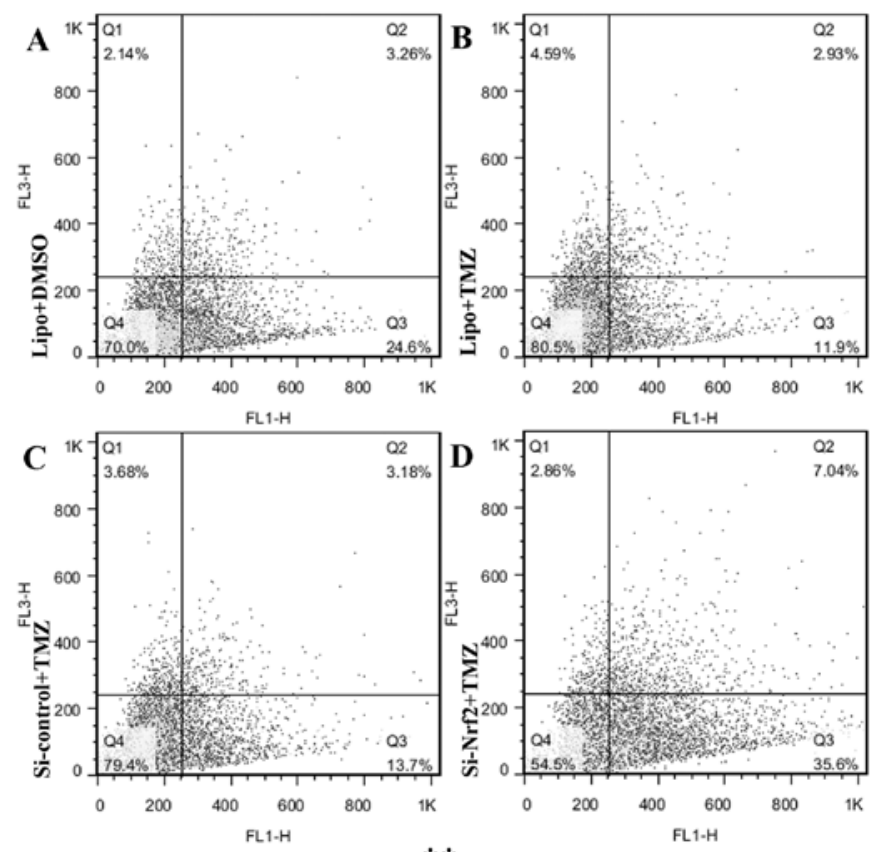

$\mathbf{E}$



Figure 6. Quantification of AVOs detected by flow cytometry with AO staining in transfected cells after $48 \mathrm{~h}$ and treated anther 3 days after TMZ $(100 \mu \mathrm{M})$. (A) Cells in group Lipo treated with DMSO for $72 \mathrm{~h}$. (B) Cells in group Lipo treated with TMZ $(100 \mu \mathrm{M})$ for 3 days. (C) Cells in group Si-control treated with TMZ $(100 \mu \mathrm{M})$ for 3 days. (D) Cells in group Si-Nrf2 treated with TMZ $(100 \mu \mathrm{M})$ for 3 days. (E) The geomean red:green fluorescence ratio showed group Si-Nrf2+TMZ had more AVOs than group Si-control+TMZ ( $\mathrm{p}=0.013)$, group Lipo+TMZ $(p=0.029)$ and group Lipo+DMSO $(p=0.001)$. Group Lipo+TMZ and group Lipo+DMSO had no significant difference. Data represent mean \pm SD values of three experiments in each group. Asterisks denotes p-value compared with Si-control group $\left({ }^{*} \mathrm{p}<0.05\right)$ and group Lipo+DMSO $\left({ }^{* *} \mathrm{p}<0.01\right)$.

role on basal autophagy, also, Nrf2 protected tumor cells from the death induced by TMZ.

The findings above illustrate the regulation of Nrf2 on autophagy induced by TMZ and its protective actions in tumor cells. Consistent with our results in U251 cells, the drug resistance of Nrf2 has also been demonstrated in a variety of experimental models (37). Transfection of Nrf2 siRNA of cisplatin-resistant human ovarian cancer SK-OV cells showed exacerbated cytotoxicity to cisplatin. Similar result was discovered in other cancer types and chemotherapeutic agents (10). In this study, knockdown of Nrf2 enhanced both the level of basal autophagy and autophagy induced by TMZ. Nevertheless, how Nrf2 regulates autophagy remains 


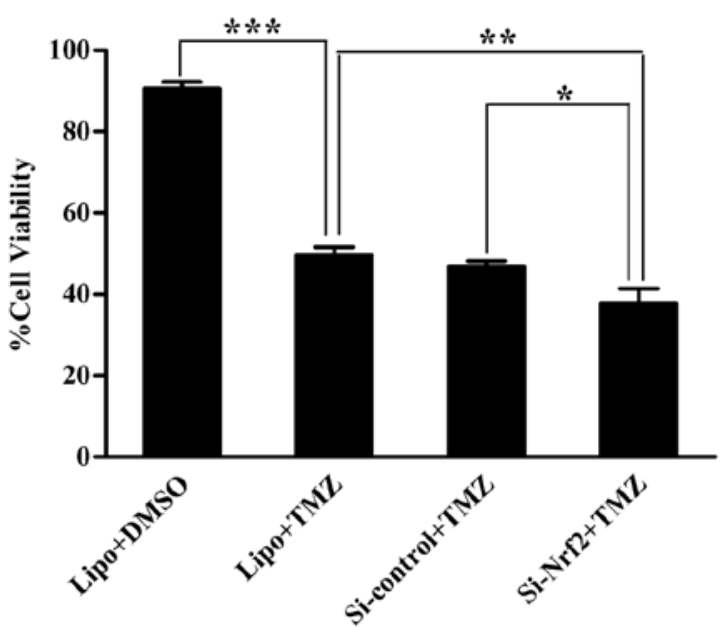

Figure 7. Cell viability analysis by CCK- 8 assay in transfected cells after $48 \mathrm{~h}$ and treated another 3 days after TMZ $(100 \mu \mathrm{M})$ for 3 days. In group Lipo, treatment of TMZ showed significantly lower cell viability than DMSO treatment $(\mathrm{p}<0.001)$. In groups treated with TMZ $(100 \mu \mathrm{M})$ for 3 days, there was more cell death in group Si-Nrf2 than that in groups Si-control $(\mathrm{p}=0.015)$ and Lipo $(p=0.007)$. There was no statistical difference between group Si-control and Lipo. Data represent mean \pm SD values of three experiments in each group. Asterisks denotes p-value between two groups. ${ }^{*} \mathrm{p}<0.05,{ }^{* *} \mathrm{p}<0.01$ group Si-Nrf2 vs. groups Si-control and Lipo, ${ }^{* * *} \mathrm{p}<0.001$, group Lipo+TMZ vs. group Lipo+DMSO.

unknown. Others have also reported that Nrf2 negatively regulated autophagy induced by anticancer redox agent MitoQ and the Nrf2-regulated enzyme NQO1 was partly responsible for adjusting the level of autophagy (35).

Recent experimental evidence showed that p62, a multidomain adapter protein, has a critical role in an oxidative stress response pathway by its direct interaction with the ubiquitin ligase adaptor Keap1, which resulted in constitutive activation of Nrf2 $(38,39)$. Another study presented evidence that p62 was an Nrf2 target gene, which created a positive feedback loop in the Nrf2-mediated transcriptional response (40). During autophagy, p62 acted not only as an adaptor protein in selective autophagy of ubiquitinated proteins, but also a substrate for degradation (41). Based on the above, we speculate that molecular foundation for possible cross-talk between Nrf2 and autophagy may be p62 or other Nrf2-targeted gene products, such as NQO1. This question and whether the protective function of Nrf2 in GBM cells after TMZ corresponds with autophagy regulated by $\mathrm{Nrf} 2$ are the issues of importance we would like to address in our future work.

In conclusion, we demonstrated for the first time that knockdown of Nrf2 enhanced autophagy induced by TMZ in U251 cells. We speculate that the combination of TMZ and the knockdown of Nrf2 may point to a novel therapeutic opportunity for GBM to enhance the antitumor effects of TMZ, which requires further studies.

\section{Acknowledgements}

This work was supported by grants of the Jiangsu Provincial Key Subject (X4200722) and the Jinling Hospital of Nanjing (Grant no. 2012035). We are thankful to Professor Shao-jun Jiang for his TEM assessment.

\section{References}

1. Germano IM, Emdad L, Qadeer ZA, Binello E and Uzzaman M: Embryonic stem cell (ESC)-mediated transgene delivery induces growth suppression, apoptosis and radiosensitization, and overcomes temozolomide resistance in malignant gliomas. Cancer Gene Ther 17: 664-674, 2010.

2. Ulasov IV, Sonabend AM, Nandi S, Khramtsov A, Han Y and Lesniak MS: Combination of adenoviral virotherapy and temozolomide chemotherapy eradicates malignant glioma through autophagic and apoptotic cell death in vivo. Br J Cancer 100: 1154-1164, 2009.

3. Stupp R, Mason WP, van den Bent MJ, et al: Radiotherapy plus concomitant and adjuvant temozolomide for glioblastoma. New Engl J Med 352: 987-996, 2005.

4. Jiang H, White EJ, Conrad C, Gomez-Manzano C and Fueyo J: Autophagy pathways in glioblastoma. Method Enzymol 453: 273-286, 2009.

5. Pan H, Wang H, Zhu L, Mao L, Qiao L and Su X: The Role of Nrf2 in migration and invasion of human glioma cell U251. World Neurosurg: Nov 7, 2011 (Epub ahead of print).

6. Zhang DD: The Nrf2-Keap1-ARE signaling pathway: The regulation and dual function of Nrf2 in cancer. Antioxid Redox Signal 13: 1623-1626, 2010.

7. Lau A, Villeneuve NF, Sun Z, Wong PK and Zhang DD: Dual roles of Nrf2 in cancer. Pharmacol Res 58: 262-270, 2008.

8. Akhdar H, Loyer P, Rauch C, Corlu A, Guillouzo A and Morel F: Involvement of $\mathrm{Nrf} 2$ activation in resistance to 5-fluorouracil in human colon cancer HT-29 cells. Eur J Cancer 45: 2219-2227, 2009.

9. Zhang J, Stevens MF and Bradshaw TD: Temozolomide: mechanisms of action, repair and resistance. Curr Mol Pharmacol 5: 102-114, 2012.

10. Wang XJ, Sun Z, Villeneuve NF, et al: Nrf2 enhances resistance of cancer cells to chemotherapeutic drugs, the dark side of Nrf2. Carcinogenesis 29: 1235-1243, 2008.

11. Levine B, Mizushima N and Virgin HW: Autophagy in immunity and inflammation. Nature 469: 323-335, 2011.

12. Klionsky DJ, Abeliovich H, Agostinis P, et al: Guidelines for the use and interpretation of assays for monitoring autophagy in higher eukaryotes. Autophagy 4: 151-175, 2008.

13. Mathew R and White E: Autophagy in tumorigenesis and energy metabolism: friend by day, foe by night. Curr Opin Genet Dev 21: 113-119, 2011.

14. Jeon SH, Kim SH, Kim Y, et al: The tricyclic antidepressant imipramine induces autophagic cell death in U-87MG glioma cells. Biochem Biophys Res Commun 413: 311-317, 2011.

15. Park EJ, Choi KS and Kwon TK: $\beta$-Lapachone-induced reactive oxygen species (ROS) generation mediates autophagic cell death in glioma U87 MG cells. Chem Biol Interact 189: 37-44, 2011.

16. Jiang H, Gomez-Manzano C, Aoki H, et al: Examination of the therapeutic potential of Delta-24-RGD in brain tumor stem cells: role of autophagic cell death. J Natl Cancer Inst 99: 1410-1414, 2007.

17. Kaza N, Kohli L and Roth KA: Autophagy in brain tumors: a new target for therapeutic intervention. Brain Pathol 22: 89-98, 2012.

18. Jakubowicz-Gil J, Langner E and Rzeski W: Kinetic studies of the effects of Temodal and quercetin on astrocytoma cells. Pharmacol Rep 63: 403-416, 2011.

19. Kanzawa T, Germano IM, Komata T, Ito H, Kondo Y and Kondo S: Role of autophagy in temozolomide-induced cytotoxicity for malignant glioma cells. Cell Death Differ 11: 448-457, 2004.

20. Natsumeda M, Aoki H, Miyahara H, et al: Induction of autophagy in temozolomide treated malignant gliomas. Neuropathology 31 : 486-493, 2011

21. Katayama M, Kawaguchi T, Berger MS and Pieper RO: DNA damaging agent-induced autophagy produces a cytoprotective adenosine triphosphate surge in malignant glioma cells. Cell Death Differ 14: 548-558, 2007.

22. Kato $\mathrm{T}$, Natsume A, Toda $\mathrm{H}$, et al: Efficient delivery of liposome-mediated MGMT-siRNA reinforces the cytotoxity of temozolomide in GBM-initiating cells. Gene Ther 17: 1363-1371, 2010.

23. Voss V, Senft C, Lang V, et al: The pan-Bcl-2 inhibitor (-)-gossypol triggers autophagic cell death in malignant glioma. Mol Cancer Res 8: 1002-1016, 2010.

24. Lefranc F: Glioblastomas are resistant to apoptosis but less resistant to the autophagic process. Bull Mem Acad R Med Belg 162: 331-338, 2007 (In French). 
25. Stepkowski TM and Kruszewski MK: Molecular cross-talk between the NRF2/KEAP1 signaling pathway, autophagy, and apoptosis. Free Radic Biol Med 50: 1186-1195, 2011

26. Gao S, Yang XJ, Zhang WG, Ji YW and Pan Q: Mechanism of thalidomide to enhance cytotoxicity of temozolomide in U251-MG glioma cells in vitro. Chin Med J 122: 1260-1266, 2009.

27. Klingelhoeffer C, Kammerer U, Koospal M, et al: Natural resistance to ascorbic acid induced oxidative stress is mainly mediated by catalase activity in human cancer cells and catalase-silencing sensitizes to oxidative stress. BMC Complement Altern Med 12: 61, 2012.

28. Lau AT, Wang Y and Chiu JF: Reactive oxygen species: current knowledge and applications in cancer research and therapeutic. J Cell Biochem 104: 657-667, 2008.

29. Bjorkoy G, Lamark T, Brech A, et al: p62/SQSTM1 forms protein aggregates degraded by autophagy and has a protective effect on huntingtin-induced cell death. J Cell Biol 171: 603-614, 2005.

30. Mizushima N and Yoshimori T: How to interpret LC3 immunoblotting. Autophagy 3: 542-545, 2007.

31. Aoki H, Kondo Y, Aldape K, et al: Monitoring autophagy in glioblastoma with antibody against isoform B of human microtubule-associated protein 1 light chain 3. Autophagy 4: 467-475, 2008.

32. Lin CJ, Lee CC, Shih YL, et al: Resveratrol enhances the therapeutic effect of temozolomide against malignant glioma in vitro and in vivo by inhibiting autophagy. Free Radic Biol Med 52: 377-391, 2012.

33. Chen H-Y and White E: Role of autophagy in cancer prevention. Cancer Prev Res 4: 973-983, 2011
34. Moreau K, Luo S and Rubinsztein DC: Cytoprotective roles for autophagy. Curr Opin Cell Biol 22: 206-211, 2010.

35. Rao VA, Klein SR, Bonar SJ, et al: The antioxidant transcription factor Nrf2 negatively regulates autophagy and growth arrest induced by the anticancer redox agent mitoquinone. J Biol Chem 285: 34447-34459, 2010.

36. Pan Q, Yang XJ, Wang HM, et al: Chemoresistance to temozolomide in human glioma cell line U251 is associated with increased activity of O6-methylguanine-DNA methyltransferase and can be overcome by metronomic temozolomide regimen. Cell Biochem Biophys 62: 185-191, 2012.

37. Cho JM, Manandhar S, Lee HR, Park HM and Kwak MK: Role of the Nrf2-antioxidant system in cytotoxicity mediated by anticancer cisplatin: implication to cancer cell resistance. Cancer Lett 260: 96-108, 2008.

38. Nezis IP and Stenmark H: p62 at the interface of autophagy, oxidative stress signaling, and cancer. Antioxid Redox Signal 17: 786-793, 2012

39. Inami Y, Waguri S, Sakamoto A, et al: Persistent activation of Nrf2 through p62 in hepatocellular carcinoma cells. J Cell Biol 193: 275-284, 2011.

40. Jain A, Lamark T, Sjottem E, et al: p62/SQSTM1 is a target gene for transcription factor NRF2 and creates a positive feedback loop by inducing antioxidant response element-driven gene transcription. J Biol Chem 285: 22576-22591, 2010.

41. Komatsu M,Kurokawa H, Waguri S, et al: The selective autophagy substrate p62 activates the stress responsive transcription factor Nrf2 through inactivation of Keap1. Nat Cell Biol 12: 213-223, 2010 . 